\title{
BMJ Open Cohort profile: effect of malaria in early pregnancy on fetal growth in Benin (RECIPAL preconceptional cohort)
}

Manfred Accrombessi, ${ }^{1,2}$ Emmanuel Yovo, ${ }^{2}$ Gilles Cottrell, ${ }^{1}$ Gino Agbota,,${ }^{1,2}$ Agnès Gartner, ${ }^{3}$ Yves Martin-Prevel, ${ }^{3}$ Nadia Fanou-Fogny, ${ }^{4}$ Diane Djossinou, ${ }^{3,4}$ Jennifer Zeitlin, ${ }^{5}$ Nicaise Tuikue-Ndam, ${ }^{1}$ Florence Bodeau-Livinec, ${ }^{5,6}$ Sandrine Houzé, ${ }^{1,7}$ Nicola Jackson, ${ }^{8}$ Paul Ayemonna, ${ }^{9}$ Achille Massougbodji, ${ }^{2}$ Michel Cot, ${ }^{1}$ Nadine Fievet, ${ }^{1}$ Valérie Briand ${ }^{1}$

To cite: Accrombessi M, Yovo E, Cottrell G, et al. Cohort profile: effect of malaria in early pregnancy on fetal growth in Benin (RECIPAL preconceptional cohort). BMJ Open

2018;8:e019014. doi:10.1136/ bmjopen-2017-019014

- Prepublication history and additional material for this paper are available online. To view these files, please visit the journal (http://dx.doi.org/10. 1136/bmjopen-2017-019014).

Received 7 August 2017 Revised 25 0ctober 2017 Accepted 10 November 2017

CrossMark

For numbered affiliations see end of article.

Correspondence to

Dr Valérie Briand;

valerie.briand@ird.fr

\section{ABSTRACT}

Purpose REtard de Croissance Intra-uterin et PALudisme (RECIPAL) is an original preconceptional cohort designed to assess the consequences of malaria during the first trimester of pregnancy, which is a poorly investigated period in Africa and during which malaria may be detrimental to the fetus.

Participants For this purpose, a total of 1214 women of reproductive age living in Sô-Ava and Akassato districts (south Benin) were followed up monthly from June 2014 to December 2016 until 411 of them became pregnant. A large range of health determinants was collected both before and during pregnancy from the first weeks of gestation to delivery. Five Doppler ultrasound scans were performed for early dating of the pregnancy and longitudinal fetal growth assessment.

Findings to date Pregnant women were identified at a mean of 6.9 weeks of gestation $(\mathrm{wg})$. Preliminary results confirmed the high prevalence of malaria in the first trimester of pregnancy, with more than $25.4 \%$ of women presenting at least one microscopic malarial infection during this period. Most infections occurred before six $w g$. The prevalence of low birth weight, small birth weight for gestational age (according to INTERGROWTH-21st charts) and preterm birth was $9.3 \%, 18.3 \%$ and $12.6 \%$, respectively.

Future plans REtard de Croissance Intra-uterin et PALudisme (RECIPAL) represents at this time a unique resource that will provide information on multiple infectious (including malaria), biological, nutritional and environmental determinants in relation to health outcomes in women of reproductive age, pregnant women and their newborns. It will contribute to better define future recommendations for the prevention of malaria in early pregnancy and maternal malnutrition in Africa. It confirms that it is possible to constitute a preconceptional pregnancy cohort in Africa and provides valuable information for researchers starting cohorts in the future.

\section{INTRODUCTION}

Malaria in pregnancy is associated with a wide range of deleterious effects in women and their offspring. In Sub-Saharan African (SSA)
Strengths and limitations of this study

- A unique cohort combining highly detailed, high-quality health-related information on the pregnancies of approximately 400 women recruited in the preconception period, with a substantial related biobank of plasmas, placental and other samples.

- Preconceptional design allowing the early identification and follow-up of pregnant women: screening of women for both microscopic and submicroscopic malaria from the first weeks of pregnancy; accurate estimation of gestational age by using early obstetrical ultrasound scan and collection of some important factors influencing fetal growth such as prepregnancy nutritional status and gestational hypertensive disorders.

- Collection of valuable information for the implementation of future preconceptional cohorts in Africa.

- High attrition in the preconceptional cohort, with a possible impact on the external validity of some findings.

- Reduced sample size at delivery due to a high proportion of spontaneous abortion, with a possible lack of power for analyses on birth outcomes.

countries, preventive strategies are based on long-lasting insecticide-treated bed nets (LLITNs) and intermittent preventive treatment in pregnancy (IPTp). IPTp consists in the administration of sulfadoxine-pyrimethamine (SP) at each antenatal care (ANC) visit from the second trimester of pregnancy onwards. ${ }^{1}$ LLITN are distributed at the first ANC visit, which generally occurs around 4-5months of pregnancy. ${ }^{2}$ Therefore, pregnant women remain unprotected or insufficiently protected during the first months of pregnancy and particularly during the first trimester. However, this period may be a high-risk period for the fetus if pregnancy-associated malarial parasites 
accumulate into the placenta during trophoblast differentiation and vascular remodelling of the uterus. ${ }^{34}$ Impaired placentation may then contribute to fetal growth restriction and low birth weight (LBW).

Few studies have investigated malaria in early pregnancy, and while some have shown an adverse effect on birth outcomes, this has not been consistent. ${ }^{5}$ In Burkina Faso and in Benin, malarial infection in the first trimester ${ }^{6}$ or before 4 to 5 months of pregnancy ${ }^{78}$ has been associated with a higher risk of LBW or a decrease in birth weight. Conversely, such association was not reported in Uganda and Malawi, ${ }^{910}$ but the number of women screened in the first trimester was low in these two studies. One of the problems in interpreting this existing literature relates to the challenges of recruiting women early in pregnancy; most of the studies were not designed to assess the consequences of malaria early in pregnancy and therefore women were generally recruited late in the first trimester, which may lead to misclassification errors and underestimation of exposure. Besides, using LBW as a proxy for fetal growth restriction (FGR) can lead to the overestimation of FGR prevalence and possible bias when estimating the effect of malaria. Finally, maternal nutrition is one of the main factors influencing fetal growth in developing countries. ${ }^{11}$ Therefore, adjusting for this factor when assessing the effect of early malaria on FGR is important. As well, a recent study has suggested a higher risk of FGR due to malaria in undernourished women. ${ }^{12}$

The REtard de Croissance Intra-uterin et PALudisme (RECIPAL) study aimed to assess the effect of malaria (both microscopic and submicroscopic) in the first trimester of pregnancy on both the mother and the fetus, with a focus on fetal growth, by following a cohort of pregnant women recruited before conception. Moreover, it also aimed at assessing the influence of the woman's nutritional status in the relationship between malaria in early pregnancy and birth outcomes.

\section{COHORT DESCRIPTION \\ Study setting}

The study cohort is currently conducted in the districts of Sô-Ava and Abomey-Calavi, located in Southern Benin (figure 1). Women's recruitment has been completed by

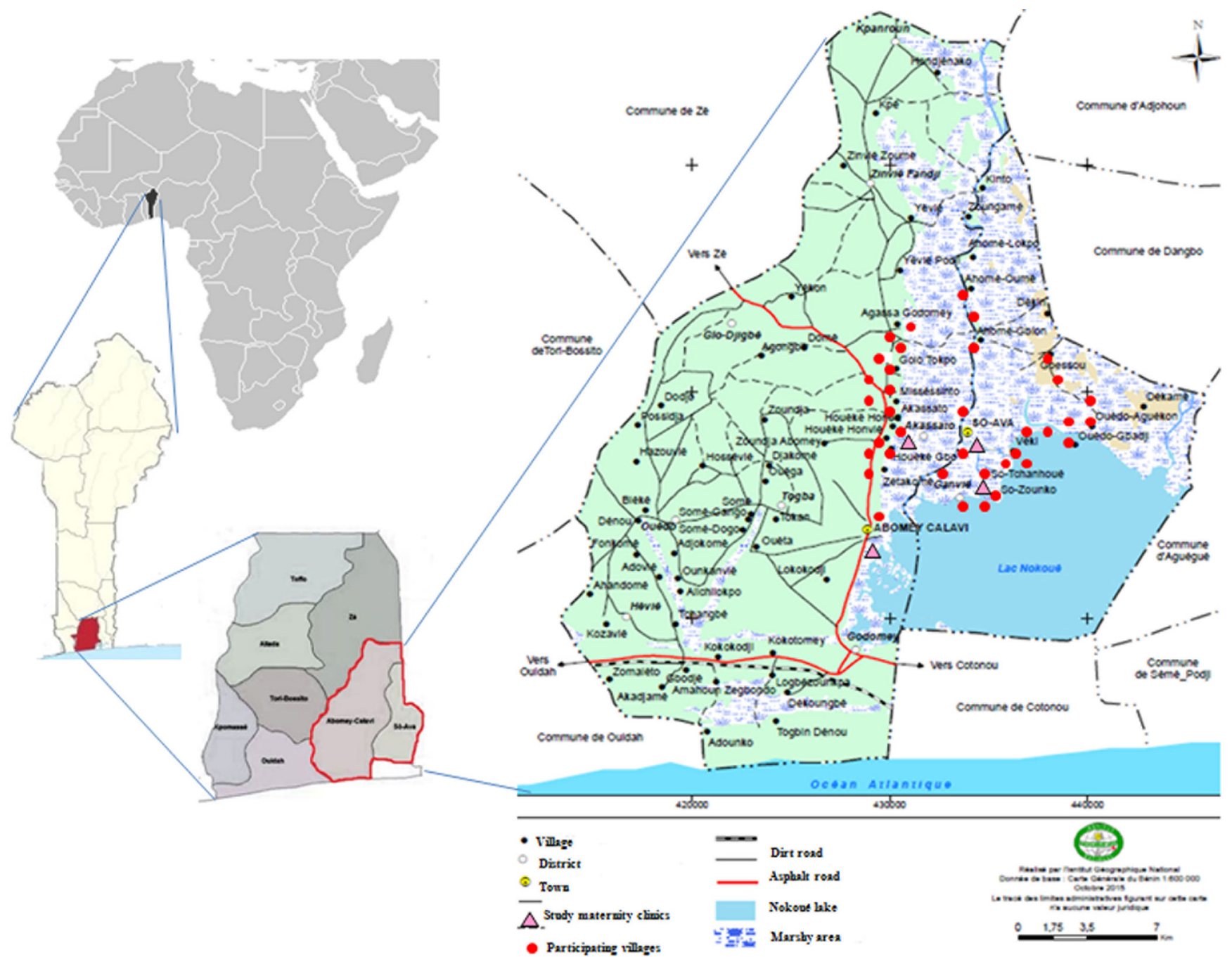

Figure 1 Geographical location of Sô-Ava and Abomey-Calavi districts and of the 35 villages of the RECIPAL study, Southern Benin, 2014-2017. RECIPAL, REtard de Croissance Intra-uterin et PALudisme. 
December 2016, and pregnancy follow-up is still ongoing. In the area, malaria is hyperendemic with a mean entomological inoculation rate of 2.1 infected anopheles bites/ person/100 nights. ${ }^{13}$ The main mosquito vectors of malaria are Anopheles gambiae ss and Anopheles funestus. ${ }^{14}$ Four subdistricts (Sô-Ava, Vekky and Houedo in Sô-Ava District and Akassato in Abomey-Calavi District) were selected for the study according to the population density and mean number of ANC visits and deliveries per month in the four main public maternity clinics of Sô-Ava and Abomey-Calavi districts. In these four subdistricts, the population size was estimated to 124994 people in 2013, including $17.4 \%$ (21 779) women of reproductive age (WRA). ${ }^{15}$ Thirty-five out of a total of 36 villages were then selected for the study according to their proximity to the maternity clinics.

\section{Study design, subject identification, recruitment and enrolment procedures}

Briefly, WRAs were recruited at community level and followed monthly for a maximum period of 24 months until becoming pregnant. They constituted the RECIPAL initial cohort. The subsample of women who became pregnant was then followed up monthly at the maternity clinic from early pregnancy to delivery. They constituted the RECIPAL final cohort.

A sample size of 466 births was estimated sufficient to evidence a two-fold OR for the association between malaria and poor birth outcomes, ${ }^{4}$ assuming a prevalence of $25 \%$ of women with microscopic malaria in the first trimester of pregnancy and 30\% of poor birth outcomes (LBW, small-for-gestational age (SGA), preterm birth (PTB) or stillbirth $)^{81617}$ with an $80 \%$ power, significant level at 0.05 . We estimated that following 2000 WRA (initial cohort) would have allowed identifying 510 pregnant women (final cohort) during a planned 18-month period for recruitment in the initial cohort (based on a total fertility rate of 200/1000/year in Benin (http://esa.un.org/unpd/wpp/ index.htm)) and allowing $15 \%$ of loss to follow-up after recruitment in the initial cohort.

After explanation of the study to the local political and administrative authorities, three concomitant procedures were used to recruit WRA. First, repeated sensitisation events were organised in each of the 35 selected villages for presenting the study to all inhabitants. If interested, women were invited to register with the head of the community and were visited at home the day after for their inclusion. That procedure constituted the main way of recruitment throughout the study. Second, leaders of the community, religious authorities and mass media were involved to help us mobilise women during public meetings (masses, women's association meetings, etc). The last way of recruitment consisted of going door to door to identify eligible and interested women with the assistance a network of community health workers (more details are provided in the online supplementary file S1).

To be included, women had met the following criteria: negative urinary pregnancy test, 18 to 45 years old, no current contraception, no previous fecundity issues, willingness to become pregnant, no planned travel for more than 2 months within the next 18 months, acceptance of RECIPAL protocol and signed written informed consent. Both oral and written communication was used for providing information on the project to the women and their husbands. The consent form was translated into the local language for women who were illiterate.

From June 2014 to December 2016, 1302 WRA were willing to participate in the study. Among them, 1214 $(93.2 \%)$ met the inclusion criteria and were recruited, $88(6.8 \%)$ were not included mainly because of a positive urinary pregnancy test at inclusion $(63.6 \%)$ or past infertility issues (14.8\%). Based on both the 2013 national census and the 2011-2012 Demographic Health Survey (DHS-IV) in Benin, we estimated that women included in the project represented $6 \%$ of the total number of WRA living in the study area. Compared with women included in Dansou et $a l$ study, ${ }^{18}$ which used individual data of a representative sample of WRA included in the Beninese DHS-IV, WRA included in RECIPAL had a higher level of poverty. The other sociodemographic characteristics such as age, education level, household wealth, gravidity and media exposure were, however, similar (online supplementary file S2).

RECIPAL study was approved by the ethics committees of the Institut des Sciences Biomédicales Appliquées (ISBA) in Benin and from the National Research Institute for Sustainable Development in France as well as by the Ministry of Health in Benin.

\section{Cohort follow-up}

\section{Preconceptional follow-up}

After enrolment, WRAs were visited at home monthly by specifically trained investigators assisted by local community health workers. Follow-up consisted mainly of recording the first day of last menstrual period (LMP) and performing a urinary pregnancy test every month. To minimise the risk of loss of confidentiality during the tracking of women for follow-up, the study team was instructed to speak only to people-including family members-to whom women have granted them permission to speak. After 12 months, women who were still followed received medical advices to help conceive, and they were invited to attend the maternity clinic for a medical examination in case of symptoms of genital infection. After 24 months, the follow-up ended and women who did not conceive were invited to attend a gynaecologist for fertility issues assessment. More details on the follow-up procedures have been provided in the online supplementary file S3 .

\section{Gestational follow-up}

Once pregnant, women were followed up at the maternity clinic for monthly ANC visits. In addition, they were encouraged to attend the maternity clinic any time in case of any symptoms. Both the maternity staff (for usual ANC follow-up and clinical examination) and study investigators (for all specific issues related to RECIPAL) were involved in women's follow-up. Throughout the study, women were offered free transport to the maternity clinic; in Sô-Ava 
District, a free river shuttle was set up. At 37weeks of gestation $(\mathrm{wg})$, women were visited at home weekly and transportation to the study maternity clinic was scheduled for delivery.

According to Beninese national recommendations, a kit including iron and folic acid tablets for 1 month, mebendazole for deworming, the first dose of SP for IPTp and a LLITN was given to each pregnant woman at the first ANC visit. The maternity staff was encouraged to administer at least three doses of IPTp from the second trimester onwards as recommended by the Beninese National Malaria Control Programme, as well as iron and folic acid supplementation throughout the pregnancy. RECIPAL women benefited from free management of any diseases related to the pregnancy-detected either as part of RECIPAL follow-up or during unscheduled visits, free ANC visits and delivery. Women infected with malaria were treated with quinine in the first trimester of pregnancy and artemether-lumefantrine from the second trimester.

\section{Infant follow-up}

The infant follow-up was not initially planned as part of the RECIPAL project. It is currently carried out as part of the SEPSIS project (bioMérieux-funding, UMR216/9198 coordination), which aims to assess immune dysfunctions associated with the risk of sepsis in preterm and growth-restricted newborns as well as in those exposed to malaria in utero. All infants born from the RECIPAL women from April 2016 have been included and followed until 3 months of age.

\section{Cohort attrition}

Among the 411 pregnant women, 207 (50.4\%) have already delivered, $16.5 \%(68 / 411)$ had a spontaneous abortion $(n=68)$ or a non-viable pregnancy $(n=2), 62 \mathrm{did}$ not complete the study because of informed consent withdrawal $(45 / 411,10.9 \%)$ or migration $(17 / 411,4.1 \%)$ and 72 women are still followed (figure 2). Spontaneous abortions, informed consent withdrawals and migrations occurred at $8.9( \pm 3.9), 13.2( \pm 8.1)$ and $14.2( \pm 8.6) \mathrm{wg}$ in average (SD), respectively. More details on the reasons for cohort attrition are provided in the online supplementary file $\mathrm{S} 4$.

Figure 2 presents the flowchart diagram of the study. Among the 1214 WRA enrolled in the initial cohort, 411 $(33.9 \%)$ became pregnant, $359(29.6 \%)$ completed the follow-up without conceiving and 444 women $(36.6 \%)$ did not complete the study mainly because of informed consent withdrawal (272/444) or migration outside the study area $(138 / 444)$ that occurred after 5.2 and 6.3 months of follow-up in average, respectively.

\section{Data collection}

Preconceptional data

Demographic, socioeconomic and household characteristics were collected at inclusion (table 1). At this occasion, screening for malaria, urinary schistosomiasis, Chlamydia trachomatis and Neisseria gonorrhoeae was performed. Haemoglobin $(\mathrm{Hb})$ and markers of inflammation levels were determined. The first day of LMP was recorded and a urinary pregnancy test (One-Step; International Holding, Germany) was performed each month. Anthropometric measurements were collected every 3 months, and dietary intakes were assessed twice during follow-up.

\section{Gestational data}

At each ANC visit, clinical, anthropometric and obstetrical data, as well as LLITN use, IPTp administration, alcohol consumption and iron, folic acid and other micronutrient supplementation intake were collected (table 2). In addition, malarial screening was performed, and proteinuria, glycosuria and urinary infection were detected using a urine dipstick test (Combi-screen;

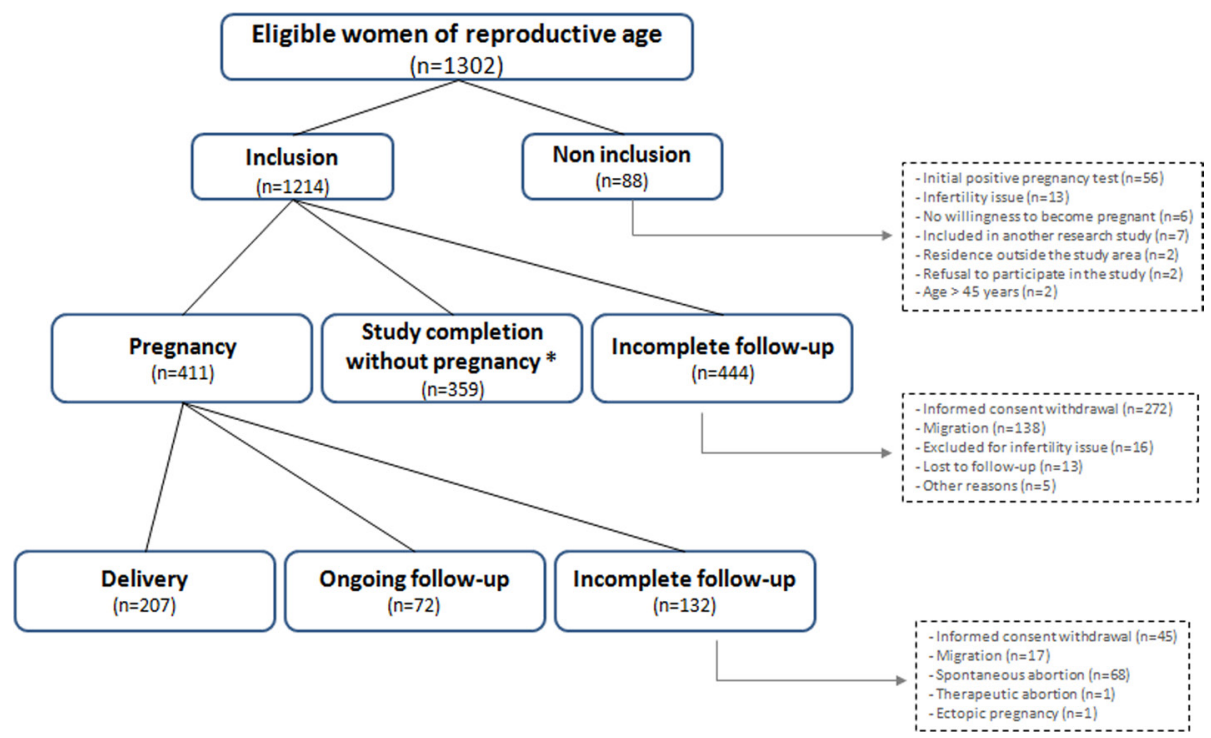

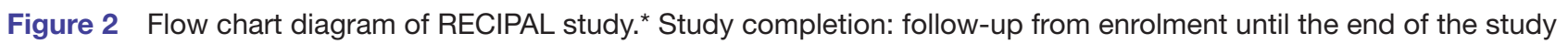
(24-month follow-up without pregnancy for women recruited before December 2014 or monthly follow-up without pregnancy for women recruited between December 2014 and December 2016), excluding consent withdrawal, migration and lost to followup. RECIPAL, REtard de Croissance Intra-uterin et PALudisme. 
Table 2 Clinical, nutritional and biological data collected during pregnancy-the RECIPAL study, 2014-2017

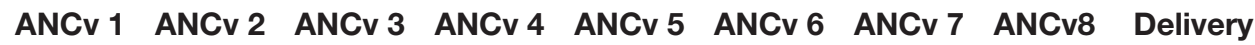

Clinical and nutritional data

Gestational age (weeks), mean (SD) $\quad 6.8 \pm 2.5 \quad 11.6 \pm 2.9 \quad 16.7 \pm 2.9 \quad 22.1 \pm 3.7 \quad 27.6 \pm 3.8 \quad 32.7 \pm 3.5 \quad 36.2 \pm 2.5 \quad 38.4 \pm 1.4 \quad 39.1 \pm 3.0$

$\begin{array}{llllllllll}\text { Medical and obstetrical history }{ }^{*} & \ddagger & \S & \S & \S & \S & \S & \S & \S & \S \\ \text { Alcohol consumption in the last } & \ddagger & \ddagger & \ddagger & \ddagger & \ddagger & \ddagger & \ddagger & \ddagger & \S\end{array}$

24 hours

\begin{tabular}{|c|c|c|c|c|c|c|c|c|c|}
\hline $\begin{array}{l}\text { Iron and folate intake in the last } \\
24 \text { hours }\end{array}$ & $\S$ & $\ddagger$ & $\ddagger$ & $\ddagger$ & $\ddagger$ & $\ddagger$ & $\ddagger$ & $\ddagger$ & $\S$ \\
\hline Use of ITN the night before the visit & $\ddagger$ & $\ddagger$ & $\ddagger$ & $\ddagger$ & $\ddagger$ & $\ddagger$ & $\ddagger$ & $\ddagger$ & $\S$ \\
\hline Axillary temperature, blood pressure & $\ddagger$ & $\ddagger$ & $\ddagger$ & $\ddagger$ & $\ddagger$ & $\ddagger$ & $\ddagger$ & $\ddagger$ & $\ddagger$ \\
\hline $\begin{array}{l}\text { Gestational age (based on LMP or } \\
\text { first US) }\end{array}$ & $\ddagger$ & $\ddagger$ & $\ddagger$ & $\ddagger$ & $\ddagger$ & $\ddagger$ & $\ddagger$ & $\ddagger$ & $\ddagger$ \\
\hline \multicolumn{10}{|l|}{ Anthropometric measurements } \\
\hline Weight & $\ddagger$ & $\ddagger$ & $\ddagger$ & $\ddagger$ & $\ddagger$ & $\ddagger$ & $\ddagger$ & $\ddagger$ & $\ddagger$ \\
\hline Mid-upper-arm-circumference & $\ddagger$ & $\S$ & $\S$ & $\ddagger$ & $\S$ & $\ddagger$ & $\S$ & $\S$ & $\S$ \\
\hline $\begin{array}{l}\text { Skinfold thickness (bicipital and } \\
\text { tricipital) }\end{array}$ & $\ddagger$ & $\S$ & $\S$ & $\ddagger$ & $\S$ & $\ddagger$ & $\S$ & $\S$ & $\S$ \\
\hline $\begin{array}{l}\text { Body composition (bioimpendance } \\
\text { analysis) }\end{array}$ & $\ddagger$ & $\ddagger$ & $\ddagger$ & $\ddagger$ & $\ddagger$ & $\ddagger$ & $\ddagger$ & $\ddagger$ & $\S$ \\
\hline Dietary 24 hours recall & $\S$ & $\ddagger$ & $\S$ & $\S$ & $\ddagger$ & $\S$ & $\ddagger$ & $\S$ & $\S$ \\
\hline \multicolumn{10}{|l|}{ Biological data } \\
\hline Blood and rhesus group & $\ddagger$ & $\S$ & $\S$ & $\S$ & $\S$ & $\S$ & $\S$ & $\S$ & $\S$ \\
\hline HIV 1 screening & $\ddagger$ & $\S$ & $\S$ & $\S$ & $\S$ & $\S$ & $\S$ & $\S$ & $\S$ \\
\hline Peripheral malaria (TBS, PCR) & $\ddagger$ & $\ddagger$ & $\ddagger$ & $\ddagger$ & $\ddagger$ & $\ddagger$ & $\ddagger$ & $\ddagger$ & $\ddagger$ \\
\hline $\begin{array}{l}\text { Placental malaria (TBS, PCR, } \\
\text { histology) }\end{array}$ & $\S$ & $\S$ & $\S$ & $\S$ & $\S$ & $\S$ & $\S$ & $\S$ & $\ddagger$ \\
\hline Cord blood malaria (TBS, PCR) & $\S$ & $\S$ & $\S$ & $\S$ & $\S$ & $\S$ & $\S$ & $\S$ & $\ddagger$ \\
\hline $\mathrm{Hb}$ level & $\S$ & $\ddagger$ & $\S$ & $\S$ & $\S$ & $\ddagger$ & $\S$ & $\S$ & $\S$ \\
\hline $\begin{array}{l}\text { Serum ferritin, sTfR, CRP, AGP, folic } \\
\text { acid levels }\end{array}$ & $\S$ & $\ddagger$ & $\S$ & $\S$ & $\S$ & $\ddagger$ & $\S$ & $\S$ & $\S$ \\
\hline Lead level & $\S$ & $\ddagger$ & $\S$ & $\S$ & $\S$ & $\ddagger$ & $\S$ & $\S$ & $\S$ \\
\hline $\begin{array}{l}\text { Urinary infection and proteinuria } \\
\text { (dipstick test) }\end{array}$ & $\ddagger$ & $\ddagger$ & $\ddagger$ & $\ddagger$ & $\ddagger$ & $\ddagger$ & $\ddagger$ & $\ddagger$ & $\S$ \\
\hline Helminthic intestinal infection (PCR) & $\ddagger$ & $\S$ & $\S$ & $\S$ & $\S$ & $\S$ & $\S$ & $\S$ & $\S$ \\
\hline Urinary schistosomiasis & $\S$ & $\S$ & $\S$ & $\S$ & $\S$ & $\S$ & $\ddagger$ & $\S$ & $\S$ \\
\hline Urine storageף & $\S$ & $\ddagger$ & $\S$ & $\ddagger$ & $\S$ & $\ddagger$ & $\S$ & $\S$ & $\S$ \\
\hline
\end{tabular}

*Sickle cell disease, diabetes or other chronic medical affection; history of preterm delivery or hypertensive disorders.

†History of cigarette smoking during pregnancy collected at delivery.

łInformation collected/assessed at each specific time point.

§Information not collected/assessed at each specific time point.

IUrine storage for further analysis.

AGP, alpha1-acid glycoprotein; ANCv, antenatal care visit; CRP, C-reactive protein; Hb, haemoglobin; ITN, insecticide-treated bed net; LMP, last menstrual period; RECIPAL, REtard de Croissance Intra-uterin et PALudisme; sTfR, soluble transferrin receptor; TBS, thick blood smear; US, ultrasound scan.

Analyticon, Germany). Dietary intakes were assessed once every trimester. Five Doppler ultrasound scans (US) were performed. A sample of venous blood was collected in the first and third trimesters for $\mathrm{Hb}$, markers of inflammation and lead levels determination.

At delivery, newborns were weighed within 1 hour after birth on an electronic digital scale with an accuracy of $2 \mathrm{~g}$ (SECA 757; SECA, Germany). Newborn's length (SECA infantometer 416, Germany) and head circumference (SECA 201, Germany) were recorded to the nearest millimetre. Malarial screening was performed on maternal venous blood, placental and cord blood (table 2). A placental biopsy was performed for malarial histopathology. Follow-up and quality control 
Table 3 Biological samples stored before conception, during pregnancy and at birth - the RECIPAL study, 2014-2017

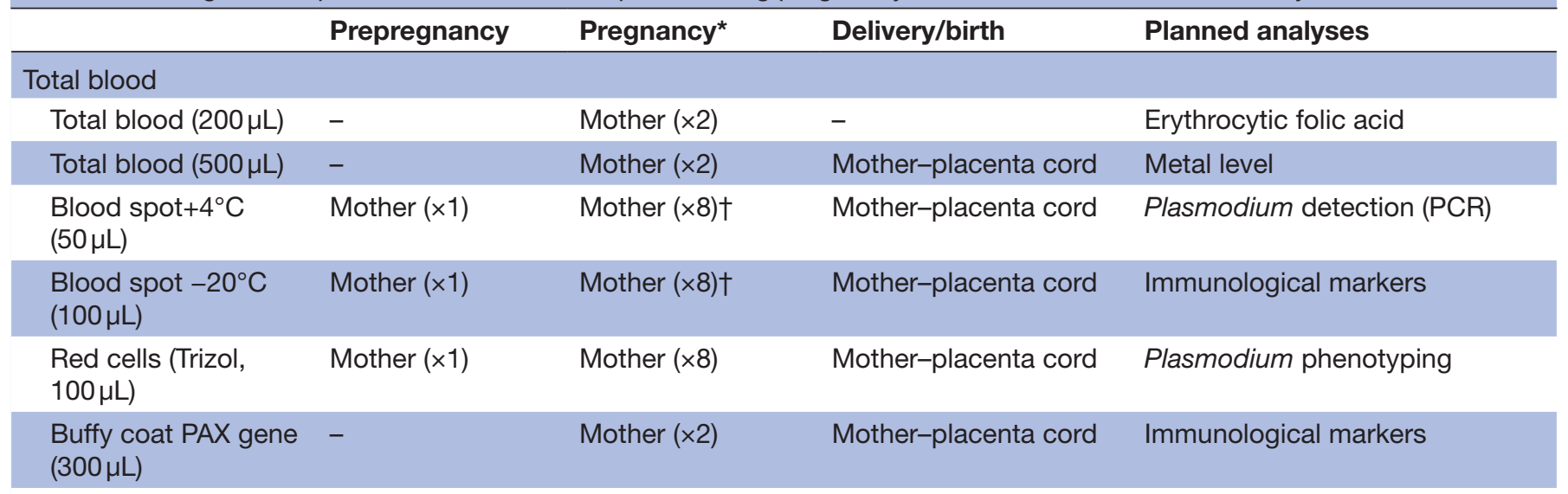

Plasma/serum

\begin{tabular}{|c|c|c|c|c|}
\hline Plasma-20 $\mathrm{C} \ddagger$ & Mother $(\times 1)$ & Mother $(\times 2)$ & Mother-placenta cord & $\begin{array}{l}\text { Micro-RNAs, cytokines and } \\
\text { chemokines }\end{array}$ \\
\hline Plasma $-80^{\circ} \mathrm{C} \ddagger$ & Mother $(\times 1)$ & Mother $(\times 2)$ & Mother-placenta cord & $\begin{array}{l}\text { Micro-RNAs, cytokines and } \\
\text { chemokines }\end{array}$ \\
\hline Serum $(100 \mu \mathrm{L})$ & Mother $(\times 1)$ & & - & $\begin{array}{l}\text { Chlamydia trachomatis and } \\
\text { Neisseria gonorrhoeae detection }\end{array}$ \\
\hline Serum $(400 \mu \mathrm{L})$ & - & Mother (×2) & & Vitamin A \\
\hline Urine (1.8mL) & Mother $(\times 2)$ & Mother $(\times 3)$ & - & Pollutant/pesticide \\
\hline Stool $\left(-80^{\circ} \mathrm{C}\right)$ & Mother $(\times 1)$ & - & - & Helminthic infection \\
\hline \multicolumn{5}{|l|}{ Placenta } \\
\hline Biopsy with formalin & - & - & Placenta & Histopathology for malaria \\
\hline
\end{tabular}

The frequency of sample collection is indicated in parentheses.

${ }^{*}$ During pregnancy, samples were collected twice (during the first and third trimester of pregnancy), three times (during each trimester) or eight times (during each ANC visit).

†In case of unscheduled visit, an additional blood spot was collected.

$\ddagger$ Quantity of plasma stored before, during pregnancy and at delivery was $200,600-800,600 \mu \mathrm{L}$, respectively.

AGP, alpha1-acid glycoprotein; ANC, antenatal care; CRP, C reactive protein; RECIPAL, REtard de Croissance Intra-uterin et PALudisme; sTfR, soluble transferrin receptor.

procedures are provided in the online supplementary file S3.

The types and origins of the biological samples collected before and during pregnancy are listed in table 3. Total blood, serum, plasma urine samples are stored in $-20^{\circ} \mathrm{C}$ or $-80^{\circ} \mathrm{C}$ freezers with a daily monitoring of the temperature.

\section{Specifics components}

\section{Malarial diagnosis}

Before conception and during pregnancy, malarial screening was performed using both microscopy (thick blood smear) and PCR. Blood smears were stained with Giemsa and parasitaemia was quantified by the Lambaréné method. ${ }^{19}$ A molecular diagnostic approach, using a combination of real-time PCR assays comprising genus-specific primers and probes for the gene encoding, the small (18S) Plasmodium rRNA and an ultrasensitive Plasmodium falciparum-specific detection system ${ }^{20}$ were used to screen samples containing Plasmodium parasites. In addition, any time during pregnancy, a rapid diagnostic test (Pf+pan rapid test SD Bioline Ag, IDA Foundation, Netherlands; BioSynex, France) was performed in case of symptoms suggestive of malaria for immediate diagnosis and treatment.

\section{Ultrasound follow-up}

The first US for dating the pregnancy was performed between 9 and $13 \mathrm{wg}$ based on LMP recorded during the preconceptional follow-up (see online supplementary file S5). The gestational age (GA) was estimated in days based on crown-rump length measurement using the Robinson's chart. ${ }^{21}$ At the end, GA estimation was based either on LMP if the difference between the two measurements (LMP/US) was less than 7 days or on US if the difference is $>7$ days. Following INTERGROWTH-21st methodology, four additional scans were performed for fetal biometry 
assessment and uterine and umbilical blood flow measurements. ${ }^{22}$ Each parameter was measured twice and then averaged; a third measure was performed in case of discrepancy between the two first measurements. A quality control was performed on $10 \%$ of the pictures.

\section{Anthropometric measurements and dietary intake assessment}

Anthropometric measurements were collected using standard procedures (tables 1 and 2). ${ }^{23}$ During household visits, women's body weight was measured with a $200 \mathrm{~g}$ precision with calibrated electronic scales (Tefal, France). At the facility level, women were weighed with a Tanita MC-780 body composition device (Tanita, Tokyo, Japan). Height was measured to the nearest millimetre with a SECA 206 (Hamburg, Germany) gauge. The left mid-upper-arm-circumference was measured with a SECA 201 (Hamburg, Germany) ergonomic circumference measuring tape. Skinfold thickness measurements were made to the nearest millimetre at the triceps and biceps sites using a Holtain (Crymyeh, UK) skinfold calliper. The set of measurements was repeated twice-by the same field investigator-and then the measurements were averaged. Body composition, assessed by bioelectrical impedance analysis method, was measured by the multifrequency body composition analyser Tanita MC-780 with a four-electrode arrangement paired on hands and feet. A quality control of anthropometric data was performed periodically by a senior research scientist in nutrition.

Dietary intakes were assessed by 24-hour recalls using the standard multiple-pass methodology. ${ }^{24}$ A comprehensive list of the most common food items and recipes was developed from a preliminary study of the diet habits of the study area. This list was used to identify foods mentioned by the women as consumed during the interview. Amounts of food consumed were estimated by household measures consisted of precalibrated utensils, food pictures of different portion sizes and monetary value of known food portions sold on the study area markets. A food composition table and recipes database are under construction for conversion of household units to gram and to determine macronutrients and micronutrients intake by the women.

\section{Characteristics of the study populations}

At enrolment, WRA were 27 years old in average (table 4). Nulligravidae represented $8.8 \%$ of the cohort. More than half of the women were anaemic, one quarter was infected with schistosomiasis and $38 \%$ had an abnormal body mass index. The prevalence of microscopic malaria was 5.7\%, with two geographical clusters in Sô-Ava and Vekky subdistricts (figure 3). The median duration of follow-up in the initial cohort was 5.9 months (mean=7.1, range: $0.49-23.7$ ). Compared with women included in the initial cohort, women who did not complete the follow-up were significantly different in terms of residence area, ethnicity, household density and schistosomiasis status (table 4).

Pregnant women were identified at a mean of $6.9 \mathrm{wg}$ and benefited from 7.5 scheduled ANC visits in average (table 4). The prevalence of microscopic malarial infection was $25.4 \%, 19.4 \%$ and $16.1 \%$ during the first, second and third trimester of pregnancy, respectively. The prevalence of SGA was $11.5 \%$ and $18.3 \%$ using Schmiegelow's and INTERGROWTH-21st charts, respectively (table 5). The proportion of PTB and LBW was $12.6 \%$ and $9.3 \%$, respectively. Overall, $29.5 \%(61 / 207)$ of newborns presented at least one poor birth outcome defined as LBW, PTB, SGA or stillbirth.

\section{FINDINGS TO DATE}

\section{Fertility and time to pregnancy}

The median time to pregnancy was 12.3 months (figure 4). The fertility rate was 5.4 pregnancies $/ 100$ personsmonth ( $95 \% \mathrm{CI}=4.9$ to 5.9$)$. The probability $(95 \% \mathrm{CI})$ of conceiving after $5,10,15$ and 20 months of follow-up were $24.5 \%$ (21.9-27.4), 42.1\% (38.6-45.9), 56.6\% (52.1-61.1) and $62.8 \%$ (57.6-68), respectively. Using multivariate survival analysis, factors associated with a lower risk of conceiving were maternal age more than 35 years, a low education level, being polygamous, living in Vekky subdistrict, long-term couple (>8years), infrequent sexual activity, overweight and urinary schistosomiasis infection (E Yovo, personal communication, 2017).

\section{Malaria in the first trimester of pregnancy}

The incidence rate of malarial infection during the first trimester was 19.7 cases per 100 persons-month (95\% CI 15.8 to 24.5 ). Using a multilevel logistic regression, we showed that women infected with malaria before conception were more likely to be infected during the first trimester (adjusted OR (aOR): 2.68, 95\% CI 1.24 to 5.78). Gestational age was also negatively correlated with malarial infection (aOR: 0.64, 95\% CI 0.41 to 0.98) (M Accrombessi, personal communication, 2017).

\section{STRENGTHS AND LIMITATIONS}

The preconceptional design-and early identification of pregnant women-allowed (1) the screening of women for malaria from the very beginning of pregnancy; (2) the accurate estimation of GA by using US before $14 \mathrm{wg}$; (3) the determination of prepregnancy nutritional status ${ }^{25}$ and hypertensive disorders screening, ${ }^{26}$ which highly influence fetal growth and (4) the minimisation of selection bias embedded in the design of women recruited at facility level. Besides, PTB and intrauterine growth restriction could be accurately assessed thanks to the precise estimation of GA and longitudinal fetal growth assessment.

In addition to malaria, RECIPAL will provide valuable information on WRA in Africa in terms of fertility and nutrition, which are seldom evaluated and may help to define new preconceptional interventions for improving maternal and child health. ${ }^{27}$ Also, it yields logistical and ethical information for the implementation of future preconceptional cohorts. 
Table 4 Characteristics of women included in the initial and final RECIPAL cohorts, Southern Benin, 2014-2017

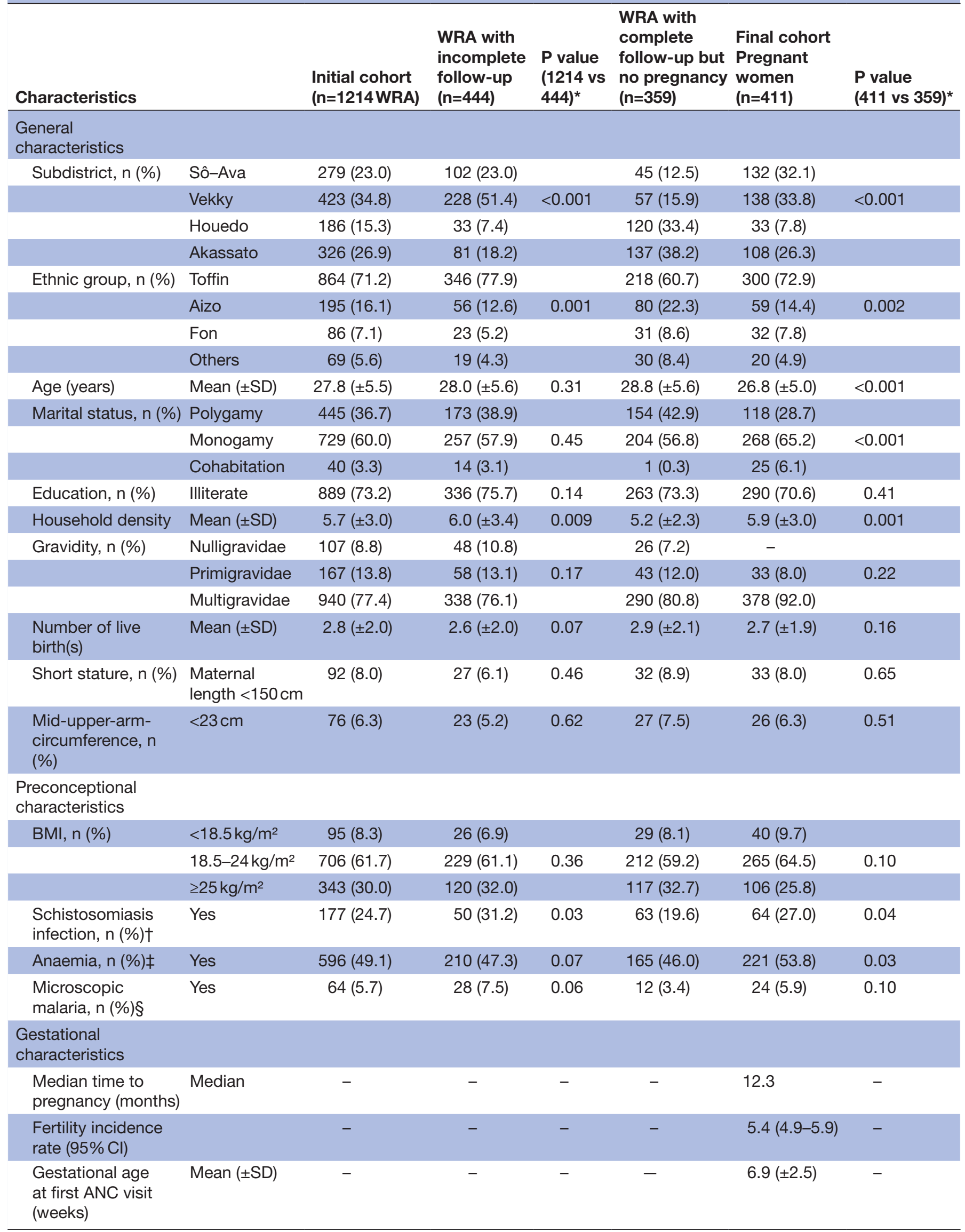


Table 4 Continued

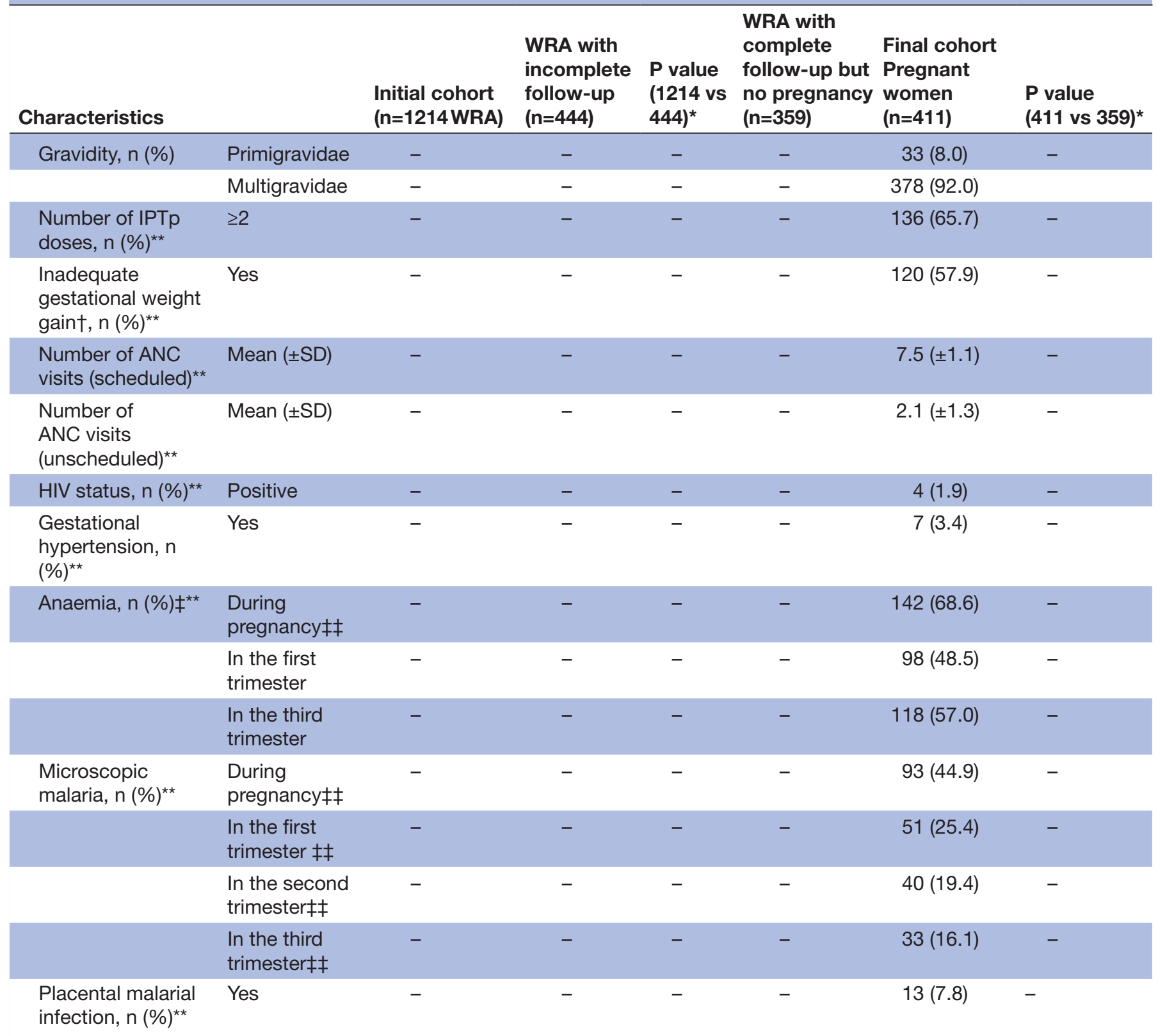

*Student's t-test and $\chi^{2}$ test were used for comparing continuous and categorical variables, respectively. †Schistosomiasis infection status before conception has been assessed in 718 WRA (initial cohort): 160 with incomplete preconceptional follow-up, 321 with complete preconceptional follow-up without pregnancy and 237 included in the final cohort.

$\ddagger$ According to WHO thresholds ( $12 \mathrm{~g} / \mathrm{dL}$ for non-pregnant women and $11 \mathrm{~g} / \mathrm{dL}$ for pregnant).

§Before conception, 78 missing and 5 missing values in the initial and final cohort, respectively. During pregnancy, 6,1 and 2 missing values at first, second and third trimester of pregnancy, respectively; 40 missing values for placental malaria.

IIncidence rate defined as the number of pregnancies/persons-month at risk.

${ }^{* *}$ Results based on the 207 women who have already delivered.

††A gestational weight gain was considered inadequate when the total weight gain during pregnancy was below to $12.5 \mathrm{~kg}, 11.5 \mathrm{~kg}, 7 \mathrm{~kg}$ and $5 \mathrm{~kg}$ in the underweight women (prepregnancy BMI $<18.5 \mathrm{~kg} / \mathrm{m}^{2}$ ), normal weighted women (prepregnancy BMI between 18.5 and 24.9 $\mathrm{kg} / \mathrm{m}^{2}$ ), overweight women (prepregnancy BMl between 25.0 and $29.9 \mathrm{~kg} / \mathrm{m}^{2}$ ) and obese women (prepregnancy BMI $\geq 30 \mathrm{~kg} / \mathrm{m}^{2}$ ) before conception, respectively.

执 At least one episode.

ANC, antenatal care; BMI, body mass index; IPTp, intermittent preventive treatment in pregnancy; RECIPAL, REtard de Croissance Intrauterin et PALudisme; WRA, women of reproductive age.

Only 107 (8\%) nulligravidae were included in the study. The main reason is that RECIPAL was implemented shortly after a study, which has recruited nulligravid WRAs living in the same area. This study aimed at assessing maternal immune responses in early pregnancy to help designing a phase 1 vaccine trial against malaria. It included 278 


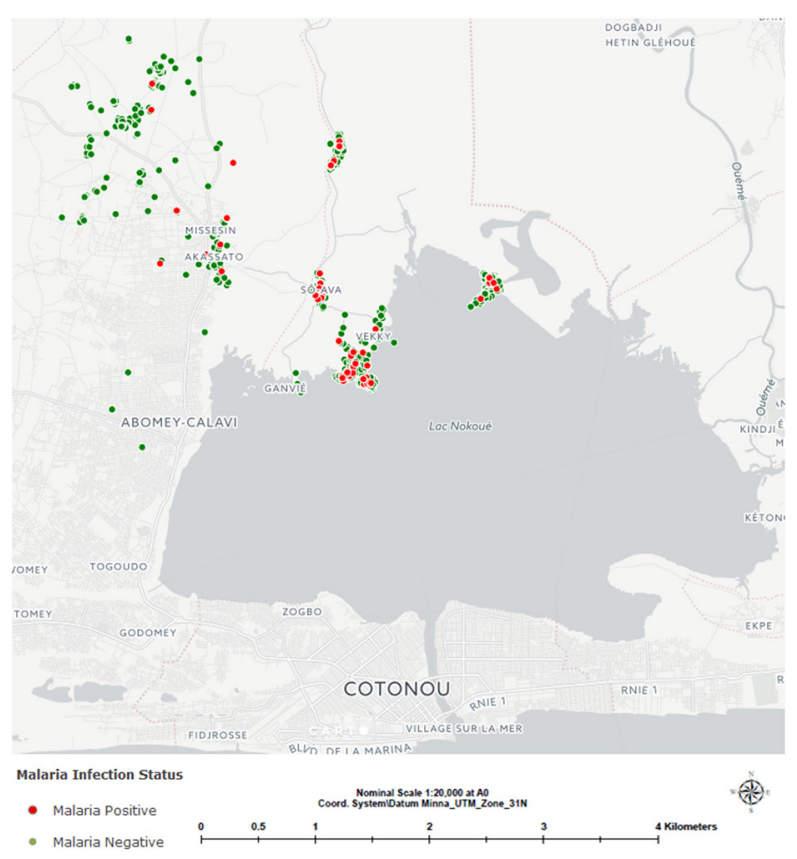

Figure 3 Malarial status (infected vs non-infected) in women of reproductive age at inclusion in RECIPAL (initial cohort), Southern Benin, 2014-2017. For online interactive map, check this link: Malaria Infection Status of Women at Inclusion of Cohort. RECIPAL, REtard de Croissance Intrauterin et PALudisme.

nulligravid WRAs, of whom 60 became pregnant. ${ }^{28}$ Both studies were conducted concomitantly for several months and women already included in the first study could not be eligible for RECIPAL. Because primigravidae were under-represented in the final RECIPAL cohort, it is likely that the prevalence of maternal conditions related to primigravidity were underestimated. Although we cannot completely exclude selection bias, it should not have strongly influenced the association between malaria in early pregnancy and FGR.
Finally, because of constraints related to the study design (long duration of the preconceptional follow-up and weariness of the women) and African sociocultural realities (rumour about blood collection, suspicion regarding medical care provided free of charge, etc), a noticeable proportion $(22 \%)$ of women withdrew their informed consent before the end of the 24-month follow-up. Overall, the initial cohort attrition was $37 \%$. Since some baseline characteristics in women who completed the preconceptional follow-up and those who did not were different, this might impact the external validity of some of results. During pregnancy, there was a high proportion $(16.5 \%)$ of spontaneous abortions, which contributed to the final cohort attrition. At the end, birth outcomes

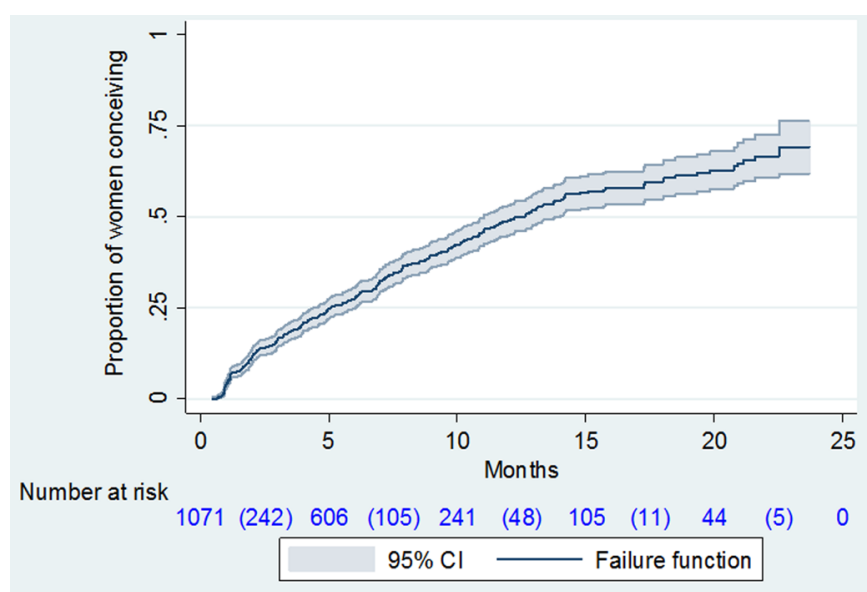

Figure 4 Kaplan-Meier failure estimate of the probability of conceiving; 1214 women of reproductive age included in the initial RECIPAL cohort, Southern Benin, 2014-2017. Probability of conceiving (solid line) and its $95 \% \mathrm{Cl}$ (grey lines). Number of pregnancy events are in parentheses. Number of censored women at 5, 10, 15,20 and 24 months were 223, 260, 88, 50 and 30, respectively. RECIPAL, REtard de Croissance Intra-uterin et PALudisme.

Table 5 Preliminary data description of newborns at birth (subsample of the cohort) - the RECIPAL study (2014-2017)

\begin{tabular}{|c|c|c|c|}
\hline Newborn characteristics* & & $\mathbf{n}$ & Mean $( \pm S D)$ or proportion (\%) \\
\hline Gender & Male & 205 & 55.2 \\
\hline Stillbirth & Per 1000 live births & 206 & 14.8 \\
\hline Small birth weight for gestational age $\dagger \neq \S$ & Yes & 191 & 11.5 \\
\hline Small birth weight for gestational age $\ddagger \S$ & Yes & 191 & 18.3 \\
\hline Birth weight $(\mathrm{g}) \ddagger$ & $<2500$ & 193 & 9.3 \\
\hline Poor birth outcomełף & Yes & 207 & 29.5 \\
\hline
\end{tabular}

*Data based on the first 207 deliveries.

†Small birth weight for gestational age: <10th percentile of birth weight for gestational age using Schmiegelow's charts. $\ddagger$ Twins excluded.

§mall birth weight for gestational age: $<10$ th percentile of birth weight for gestational age using INTERGROWTH-21st charts.

IStillbirth, preterm birth, small birth weight for gestational age (using INTERGROWTH-21st charts) or low birth weight.

RECIPAL, REtard de Croissance Intra-uterin et PALudisme. 
could be evaluated in only $68 \%$ of women, with a possible lack of power for some upcoming analyses.

\section{CONCLUSION}

If the deleterious effects of malaria in the first trimester of pregnancy are confirmed, our results may argue in favour of starting preventive measures from the very beginning of pregnancy or even before pregnancy. Artemisinin-based combination therapies have been shown to be safe during the first trimester of pregnancy and, therefore, may be a good option for the replacement of SP. ${ }^{29}$ Besides, a vaccine against pregnancy-associated malarial parasites, which could elicit protective immunity prior to pregnancy to best protect pregnant women during early pregnancy may be proposed as a complementary strategy. Such a vaccine is currently under evaluation. ${ }^{30}$

\section{Author affiliations}

${ }^{1}$ UMR216-MERIT, French National Research Institute for Sustainable Development (IRD), Université Paris Descartes, Paris, France

${ }^{2}$ Centre d'Etude et de Recherche sur le Paludisme Associé à la Grossesse et à I'Enfance (CERPAGE), Cotonou, Benin

${ }^{3}$ UMR204-Nutripass, French National Research Institute for Sustainable

Development (IRD), Université de Montpellier, Montpellier, France

${ }^{4}$ Ecole de Nutrition et des Sciences et Technologies Alimentaires (ENSTA),

Faculté des Sciences Agronomiques, Université d'Abomey-Calavi, AbomeyCalavi, Benin

${ }^{5}$ Obstetrical, Perinatal and Pediatric Epidemiology Research Team, Centre for Epidemiology and Biostatistics (U1153-EPOPé), National Institute for Health and Medical Research (INSERM), Université Paris Descartes, Paris, France

${ }^{6}$ Département Méthodes Quantitatives en Santé Publique, Ecole des Hautes Etudes en Santé Publique (EHESP), Université de Rennes, Rennes, France

${ }^{7}$ Laboratoire de Parasitologie, CNR du Paludisme, AP-HP, Hôpital Bichat, Paris, France

${ }^{8}$ Department of Obstetrics and Gynaecology, Oxford University, Oxford, UK ${ }^{9}$ Service de Gynécologie et Obstétrique, Centre Hospitalier Universitaire d'AbomeyCalavi, Abomey-Calavi, Benin

Acknowledgements We are extremely grateful to all families who took part in this study, the midwifes, nurses and community health workers for recruiting and following them and the whole RECIPAL team, including research scientists, engineers, technicians and managers. We also thank Seun Egbinola for study area mapping.

Collaborators The "Mother and child face to tropical infections" research unit (MERIT), French National Research Institute for Sustainable Development (IRD)/Paris Descartes University was the promoter of the study. RECIPAL was a collaborative project between MERIT, another IRD research unit (Nutripass, Montpellier), EPOPé research team (Inserm), the Ecole des Hautes Etudes en Santé Publique (EHESP) and two Beninese collaborators from Abomey-Calavi University (the Faculty of Health Sciences and the Faculty of Agronomy Sciences).

Contributors Project management: MA, NF, AM, MC and VB (principal investigator). Field, epidemiology and data collection: MA, EY, GC, GA, PA, NF and VB. Nutritional data collection: YM-P, AG, NF-F, DD, MA and EY. Biology and molecular analyses: NF, NT-N and SH. Obstetrical data collection: MA, EY, NJ and VB.Statistical analysis: MA, GC, MC, JZ, FB-L, YM-P, AG, NF-F, DD and VB. Manuscript writing: MA, GA, GC, AG, YM-P, NFF, DD, JZ, NTM, FB-L, SH, MC, NF and VB.

Funding This study was supported by the French National Research Agency (ANR, ANR-13-JSV1-0004, grant 2013) and the Fondation de France (no: 00074147, grant 2017). MA was funded by the Réseau doctoral de l'Ecole des Hautes Etudes en Santé Publique (EHESP) for PhD scholarship and received a prize from the Fondation des Treilles (http://www.les-treilles.com/en/).

Competing interests None declared.

Patient consent Obtained.
Ethics approval The ethics committees of the Institut des Sciences Biomédicales Appliquées (ISBA) and the Ministry of Health in Benin.

Provenance and peer review Not commissioned; externally peer reviewed.

Data sharing statement The researchers associated with this study are open for the sharing and reuse of the data, but an end-user data use agreement will be required for accessing the data. Collaborations are encouraged, although data sets are not currently publicly available. Any researcher interested in exploring RECIPAL data should contact directly the principal investigator, Valerie Briand (email: valerie. briand@ird.fr)

Open Access This is an Open Access article distributed in accordance with the Creative Commons Attribution Non Commercial (CC BY-NC 4.0) license, which permits others to distribute, remix, adapt, build upon this work non-commercially, and license their derivative works on different terms, provided the original work is properly cited and the use is non-commercial. See: http://creativecommons.org/ licenses/by-nc/4.0/

(C) Article author(s) (or their employer(s) unless otherwise stated in the text of the article) 2018. All rights reserved. No commercial use is permitted unless otherwise expressly granted.

\section{REFERENCES}

1. WHO Malaria Policy Advisory Committee and Secretariat. Malaria Policy Advisory Committee to the WHO: conclusions and recommendations of eighth biannual meeting (September 2015). Malar J 2016;15:117.

2. Crawley J, Hill J, Yartey J, et al. From evidence to action? Challenges to policy change and programme delivery for malaria in pregnancy. Lancet Infect Dis 2007;7:145-55.

3. Umbers AJ, Aitken EH, Rogerson SJ. Malaria in pregnancy: small babies, big problem. Trends Parasitol 2011;27:168-75.

4. Griffin JB, Lokomba V, Landis SH, et al. Plasmodium falciparum parasitaemia in the first half of pregnancy, uterine and umbilical artery blood flow, and foetal growth: a longitudinal Doppler ultrasound study. Malar J 2012;11:319.

5. Huynh BT, Cottrell G, Cot M, et al. Burden of malaria in early pregnancy: a neglected problem? Clin Infect Dis 2015;60:598-604.

6. Valea I, Tinto H, Drabo MK, et al. An analysis of timing and frequency of malaria infection during pregnancy in relation to the risk of low birth weight, anaemia and perinatal mortality in Burkina Faso. Malar $J$ 2012;11:71.

7. Cottrell G, Mary JY, Barro D, et al. The importance of the period of malarial infection during pregnancy on birth weight in tropical Africa. Am J Trop Med Hyg 2007;76:849-54.

8. Huynh BT, Fievet N, Gbaguidi G, et al. Influence of the timing of malaria infection during pregnancy on birth weight and on maternal anemia in Benin. Am J Trop Med Hyg 2011;85:214-20.

9. De Beaudrap P, Turyakira E, White LJ, et al. Impact of malaria during pregnancy on pregnancy outcomes in a Ugandan prospective cohort with intensive malaria screening and prompt treatment. Malar $J$ 2013;12:139.

10. Kalilani-Phiri L, Thesing PC, Nyirenda OM, et al. Timing of malaria infection during pregnancy has characteristic maternal, infant and placental outcomes. PLoS One 2013;8:e74643.

11. Osrin D, de L Costello AM. Maternal nutrition and fetal growth: practical issues in international health. Semin Neonatol 2000;5:209-19.

12. Cates JE, Unger HW, Briand V, et al. Malaria, malnutrition, and birthweight: a meta-analysis using individual participant data. PLOS Med 2017:14:e1002373.

13. Djènontin A, Bio-Bangana $S$, Moiroux N, et al. Culicidae diversity, malaria transmission and insecticide resistance alleles in malaria vectors in Ouidah-Kpomasse-Tori district from Benin (West Africa): A pre-intervention study. Parasit Vectors 2010;3:83.

14. Corbel V, N'Guessan R, Brengues C, et al. Multiple insecticide resistance mechanisms in Anopheles gambiae and Culex quinquefasciatus from Benin, West Africa. Acta Trop 2007;101:207-16.

15. Institut National de la Statistique et de l'Analyse Economique. Fourth (2013) general census of population and habitation in Benin. 2015.

16. Briand V, Saal J, Ghafari C, et al. Fetal growth restriction Is associated with malaria in pregnancy: a prospective longitudinal study in Benin. $J$ Infect Dis 2016;214:417-25.

17. Unger HW, Ome-Kaius M, Karl S, et al. Factors associated with ultrasound-aided detection of suboptimal fetal growth in a malariaendemic area in Papua New Guinea. BMC Pregnancy Childbirth 2015;15:83. 
18. Dansou J, Adekunle AO, Arowojolu AO. Factors associated with antenatal care services utilisation patterns amongst reproductive age women in Benin Republic: an analysis of 2011/2012 benin republic's demographic and health survey data. Niger Postgrad Med J 2017;24:67-74.

19. Planche T, Krishna S, Kombila M, et al. Comparison of methods for the rapid laboratory assessment of children with malaria. Am J Trop Med Hyg 2001;65:599-602.

20. Hofmann N, Mwingira F, Shekalaghe S, et al. Ultra-sensitive detection of Plasmodium falciparum by amplification of multi-copy subtelomeric targets. PLoS Med 2015;12:e1001788.

21. Robinson HP, Fleming JE. A critical evaluation of sonar "crown-rump length" measurements. Br J Obstet Gynaecol 1975;82:702-10.

22. Papageorghiou AT, Sarris I, loannou C, et al. Ultrasound methodology used to construct the fetal growth standards in the INTERGROWTH-21 ${ }^{\text {st }}$ Project. Int J Gynaecol Obstet 2013;120(Suppl 2):27-32.

23. Lohman T, Roche A, Martorell R. Anthropometric standardisation reference manual. Champaign, IL: Human Kinetics, 1998.

24. Gibson R, Gibson RS. Principles of nutritional assessment (second edition). Oxford, UK: Oxford University Press, 2005.
25. Kramer MS. The epidemiology of adverse pregnancy outcomes: an overview. J Nutr 2003;133:1592S-6.

26. Ndao CT, Dumont A, Fievet N, et al. Placental malarial infection as a risk factor for hypertensive disorders during pregnancy in Africa: a case-control study in an urban area of Senegal, West Africa. Am J Epidemiol 2009;170:847-53.

27. Persson LÅ, Arifeen S, Ekström EC, et al. Effects of prenatal micronutrient and early food supplementation on maternal hemoglobin, birth weight, and infant mortality among children in Bangladesh: the MINIMat randomized trial. JAMA 2012;307:2050-9.

28. Gbédandé K, Fievet N, Viwami F, et al. Clinical development of a VAR2CSA-based placental malaria vaccine PAMVAC: quantifying vaccine antigen-specific memory $B \&$ T cell activity in Beninese primigravidae. Vaccine 2017;35:3474-81.

29. Moore KA, Simpson JA, Paw MK, et al. Safety of artemisinins in first trimester of prospectively followed pregnancies: an observational study. Lancet Infect Dis 2016;16:576-83.

30. Tuikue-Ndam N, Deloron P. Developing vaccines to prevent malaria in pregnant women. Expert Opin Biol Ther 2015;15:1173-82. 